116

\section{Gastric Polyps: Where Do We Stand?}

Anibal Ferreira, MD, João Soares, MD, Ana Caetano, MD, Carla Rolanda, MD, PhD, Artur Machado, MD, Raquel Gonçalves, MD. Gastroenterology, Hospital São Marcos, Braga, Portugal.

Purpose: 1-4\% of patients undergoing upper GI endoscopy have gastric polyps. These lesions may be true epithelial polyps, heterotopias, lymphoid tissue, or stromal lesions. Hyperplastic polyps are the most frequently encountered and occur in both sexes, being more common in the seventh decade of life. The objective was to describe the endoscopic and histopathological characteristics of gastric polyps and establish connections with demographic criteria of our population.

Methods: Retrospective analysis of a total of 106 polyps removed with polypectomy snare in the years 2008 and 2009, using endoscopy reports and histopathology results of the lesions. Epidemiologic data was obtained from patient files and was statistically processed with Excel $2007^{\oplus}$ and SPSS $(\mathrm{v} 16.0)^{\oplus}$.

Results: $80.2 \%$ were hyperplastic, $9.4 \%$ true adenomas, and $5.7 \%$ were fundic glands. Hyperplastic polyps average size was $11.5 \mathrm{~mm}$ with preferential location in the antrum (52.9\%). Most adenomas were sessile (80\%) and were located preferentially in the body (50\%), with an average size of $8.9 \mathrm{~mm}(60 \%$ of those showed high-grade dysplasia). The majority of individuals were infected with Helicobacter pylori and less than a third was medicated with a PPI at the time of polypectomy. There were no major differences between sexes and age distribution. The authors also present the results of univariate and multivariate analysis of epidemiological associations.

Conclusion: Most polyps were hyperplastic, confirming the association with areas of high prevalence of Helicobacter pylori infection.2 The small number of adenomas limits statistical results, but clearly a large portion of them had high grade dysplasia in relation with other epidemiological aspects of our population.

\section{7}

Quadruple Therapy Using a 3-in-1 Capsule of Bismuth Subcitrate Potassium, Metronidazole, and Tetracycline with Omeprazole Compared to Triple Therapy for the Eradication of Helicobacter pylori 2010 Presidential Poster

Paul Moayyedi, MB, ChB, ${ }^{1}$ Franco Bazzoli, $M D,{ }^{2}$ Francis Megraud, $M D,{ }^{3}$ Monique Giguere, $P h D^{4}$. 1. McMaster University, Hamilton, ON, Canada; 2. Universita di Bologna, Bologna, Italy; 3. Universite Victor Segalen Bordeaux 2, Bordeaux, France; 4. Axcan Pharma, Inc., Mont-Sant-Hilaire, QC, Canada.

Purpose: Although proton pump inhibitor triple therapy is recommended as first line treatment of $H$. pylori, eradication rates $<80 \%$ have been recently reported. Bismuth containing quadruple therapy is an alternative. The efficacy of these 2 approaches was evaluated by re-analyzing 2 randomized controlled trials (RCTs).

Methods: One N. American RCT comparing omeprazole plus a 3-in-1 capsule of bismuth subcitrate potassium, metronidazole, and tetracycline (OBTM) vs. omeprazole, amoxicillin and clarithromycin (OAC) each given for 10 days was combined with a European RCT comparing OBTM (10 days) vs. OAC (7 days). Both RCTs evaluated therapy in patients with upper GI symptoms, positive 13C urea breath test (UBT), a rapid urease test with at least 1 positive test from histology and culture. H. pylori eradication was confirmed by 2 negative UBTs 6 and 10 weeks post therapy. The impact of sex, age, race, presence of peptic ulcer disease (PUD), duration of OAC therapy, and pretreatment $H$. pylori clarithromycin and metronidazole resistance was evaluated on efficacy in univariate and multivariate analyses. All data were analyzed on an intention to treat basis.

Results: 299 patients were enrolled in the N. American RCT with eradication rates in OBMT arm of 126/147 (86\%; 95\% CI=79\%-91\%) and 122/152 $(80 \% ; 95 \% \mathrm{CI}=73 \%-86 \%)$ in OAC arm. 440 patients were enrolled in the
European RCT with eradication rates of $174 / 218$ (80\%; 95\% CI=74\%-85\%) with 10d OBTM and 123/222 (55\%; 95\% CI=49\%-62\%) with 7d OAC. There were no predictors of treatment failure in OBTM arms in univariate or multivariate analysis including $H$. pylori metronidazole resistant strains $(81 \%$ versus $86 \%, p=0.23)$. OAC was less successful in patients without PUD vs. those with PUD (eradication rate $60 \%$ vs. $77 \%$ respectively; $\mathrm{p}=0.003$ ), in those taking $7 \mathrm{~d}$ vs. $10 \mathrm{~d}$ of therapy ( $80 \%$ vs. $55 \%$; $\mathrm{p}<0.0001)$ and in clarithromycin resistant strains ( $12 \%$ vs. $78 \%, p<0.0001)$. These factors remained significant in a logistic regression model adjusting for all factors outlined above. In a logistic regression model combining OBTM and $\mathrm{OAC}$ groups adjusting for all factors plus an interaction term for clarithromycin resistance and treatment regimen, clarithromycin resistance $(p=0.004)$ was an independent predictor of treatment failure whereas therapy with OBTM was an independent predictor of treatment success $(\mathrm{p}=0.003)$.

Conclusion: OBTM appears less susceptible to influence of $H$. pylori resistance to antibiotics and other factors in determining efficacy and overall may be a better first line choice in the treatment of $H$. pylori infection.

Disclosure: Dr Moayyedi, Advisory Board Member, Axcan Pharma; Dr Bazzolo, Advisory Board Member, Axcan Pharma; Prof Megraud, Advisory Board Member and Dr Giguere, Employee, Axcan Pharma.

This research was supported by an industry grant from Axcan Pharma.

\section{8}

Effect of Yoga (Shankh Prakshalana) on Liquid Gastric Emptying Pattern Measured By Real Time Ultrasound

Vijaypal Arya, MD, ${ }^{1}$ Ashok Valluri, MD, MS, MPH, ${ }^{1}$ Jitin Patel, $M S,{ }^{1}$ Jeffery Fischbein, MD, ${ }^{2}$ Larissa Davidowa, RDMS ${ }^{2}$. 1. Vijaypal Arya MD PC, Middle Village, NY; 2. Wyckoff Heights Medical Center, Brooklyn, NY.

Purpose: Originated in India, more than 2500 years ago, Yoga is now in widespread practice throughout the United States. Shankh Prakshalana is a Yogic process intended to clean the bowel using lukewarm saline water and specific sets of yoga postures (Asanas). Using this Yogic method to clean the colon, we reported the efficacy similar to Polyethylene glycol based colon prep with an average prep time of 101 minutes. We postulate that Yoga postures in Shankh Prakshalana, modify the gastric configuration and gastric emptying leading to faster gastric emptying and faster colon lavage.

Methods: After six hours of fasting, gastric emptying pattern analysis was done in 10 ( 5 males, 5 females) healthy volunteers. The protocol was approved by IRB at WHMC. All ultrasound measurements were done by a well trained technician. The volunteers drank $480 \mathrm{~mL}$ of lukewarm saline in a bolus form (1-2 minutes). Gastric antral ultrasounds were done - Baseline, immediately after intake and at 10,20 and 30 minutes interval. On a different day, the process was repeated with Asanas performed in between intervals. Return to the baseline surface area and/or appearance of gas shadow in the antrum were used as a secondary guideline for complete emptying. The results were expressed as percentage change in the surface area of gastric antrum from antral surface area measured at immediately after intake.

Results: The percentage decrease in the surface area of gastric antrum at 10,20 and 30 minutes from immediately after intake were - (minus) 1.44, 32.83, 51.14 without Asanas and 23.47, 42.26, 58.69 with Asanas. The major difference in gastric antral surface area was evident at first 10 minutes - increased in non Yoga group as compared to decrease in the Yoga group. Gastric emptying patterns in males was faster than females. One patient showed paradoxical effect and is not included in this analysis.

Conclusion: The Yoga allowed normal saline to pass gastric antrum without accumulation, leading to faster emptying. Bolus intake of normal saline itself makes the gastric emptying process faster (first order kinetics) and it was further enhanced by these Asanas. This may explain the faster colon preparation in bolus lukewarm saline Yogic method of colon cleaning. 